\title{
Transient analysis of FGM plates bending under thermal loading: comparative study within classical and generalized thermoelasticity
}

\author{
Ladislav Sator ${ }^{1, *}$, Vladimir Sladek ${ }^{1}$, and Jan Sladek ${ }^{1}$ \\ ${ }^{1}$ Institute of Construction and Architecture, Slovak Academy of Sciences, 84503 Bratislava, Slovakia
}

\begin{abstract}
In the classical thermoelasticty, there is coupling between thermal and elastic fields, in general, but the elastic and thermal excitations spread according to physically different laws. In classical thermodynamics, the temperature change propagates according to diffusion law. However, the heat pulses at low temperature propagate evidently with a finite velocity in view of waves similar to spreading of elastic excitations. A great effort has been spent in this matter and there are two main nonclassical theories: Lord-Shulman theory and Green-Lindsay theory. In this paper, we compare the response of thin FGM plates under thermal load within the classical and non-classical theories of thermoelasticity. The variable material properties of plate (such as the Young's modulus, thermal expansion coefficient, etc.) are allowed to be continuous functions of the position. The strong form meshless formulations for solution of considered initial-boundary value problems is developed in combination with Moving Least Squares (MLS) approximation scheme. The response of FGM plates on thermal loading is studied via numerical simulations with focusing on comparison of results obtained within the classical and generalized thermoelasticity. The numerical results concern also the parametric study of influence of gradation of material coefficients on bending of FGM plates
\end{abstract}

Keywords: transient thermoelasticity, functionally graded material, Kirchhoff-Love plate theory, strong formulation, meshless approximation

\section{Introduction}

Thermal stresses, especially at the interface between two different materials, often represent a significant factor in the failure of laminated composite structures. Thus, there is a need to accurately predict thermal stresses in composite structures [9]. Simultaneously, this is also a strong motivation to replace laminated plate structures by FGM ones if possible. Therefore the study of behaviour of FGM plates under thermal loadings has become attractive, what can be confirmed by a huge amount of papers devoted to thermoelastic analyses with using various plate bending theories for FGM plates under static and/or

\footnotetext{
*Corresponding author: ladislav.sator@savba.sk

Reviewers: Radim Halama, Janusz Mielniczuk
} 
dynamic loadings [6-7]. In the classical thermoelasticity, the temperature field is coupled with the elastic fields, in general, but the temperature change does not propagate in a wave form [1]. However, the heat pulses at low temperature propagate with a finite velocity like waves. Such a behaviour is described within Lord-Shulman theory of generalized thermoelasticity [10]. In this paper, the bending of thin FGM plates under thermal load is considered within the classical and generalized theory of thermoelasticity. The governing equations which are given by the 4th order partial differential equations (PDE) are decomposed into the 2nd order PDEs in order to overcome the inaccuracy of approximation of high order derivatives of field variables. The strong form meshless formulations for solution of thin plate bending problem is developed in combination with Moving Least Squares (MLS) approximation scheme [2]. The attention is paid to the numerical investigation of the influence of several parameters of gradations of material coefficients on the deflection of the plate.

\section{Governing equations}

Let us consider FGM plates with the power-law transversal gradation of Young's modulus, thermal expansion, heat conduction coefficients, specific heat capacity and mass density as:

$$
\begin{array}{ll}
E\left(x_{3}\right)=E_{0} E_{V}\left(x_{3}\right), & E_{V}\left(x_{3}\right)=1+\zeta\left(\frac{1}{2} \pm \frac{x_{3}}{h}\right)^{p} \\
\alpha\left(x_{3}\right)=\alpha_{0} \alpha_{V}\left(x_{3}\right), & \alpha_{V}\left(x_{3}\right)=1+\xi\left(\frac{1}{2} \pm \frac{x_{3}}{h}\right)^{r} \\
k\left(x_{3}\right)=k_{0} k_{V}\left(x_{3}\right), \quad k_{V}\left(x_{3}\right)=1+\omega\left(\frac{1}{2} \pm \frac{x_{3}}{h}\right)^{s} \\
c\left(x_{3}\right)=c_{0} c_{V}\left(x_{3}\right), \quad c_{V}\left(x_{3}\right)=1+\chi\left(\frac{1}{2} \pm \frac{x_{3}}{h}\right)^{q} \\
\rho\left(x_{3}\right)=\rho_{0} \rho_{V}\left(x_{3}\right), \quad \rho_{V}\left(x_{3}\right)=1+\varepsilon\left(\frac{1}{2} \pm \frac{x_{3}}{h}\right)^{g} .
\end{array}
$$

where $\zeta, \xi, \omega, \chi, \varepsilon$ and $p, r, s, q, g$ are the values of the level and exponent of the powerlaw gradation, respectively.

Before writing the governing equations in Kirchhoff - Love theory (KLT) of thin plates for thermoelastic initial-boundary value problems (IBVP) we define the dimensionless coordinates and time variable as

$$
x_{\beta}^{*}:=\frac{x_{\beta}}{L}, x_{3}^{*}:=\frac{x_{3}}{h_{0}}=h^{*}(\mathbf{x}) z, h(\mathbf{x})=h_{0} h^{*}(\mathbf{x}), t^{*}:=\frac{t}{T}
$$


with $L, h_{0}, T$ being the characteristic in-plane dimension, plate thickness and time interval. The dimensionless field variables in the KLT (in-plane displacements, deflections, temperature) within classical thermoelasticity are introduced as:

$$
u_{\beta}^{*}(\mathbf{x}, t):=\frac{u_{\beta}(\mathbf{x}, t)}{h_{0}}, \quad w^{*}(\mathbf{x}, t):=\frac{w(\mathbf{x}, t)}{h_{0}}, \vartheta_{a}^{*}(\mathbf{x}, t):=\frac{\vartheta_{a}(\mathbf{x}, t)}{\theta_{0}},
$$

where we have omitted the superscript $*$ in coordinates and time variable and three fields $\vartheta_{a}(\mathbf{x}, t)$ occur in the truncated expansion of the temperature w.r.t. the $z$-coordinate: $\theta^{*}(\mathbf{x}, z, t) \approx 1+\vartheta_{0}^{*}(\mathbf{x}, t)+z \vartheta_{1}^{*}(\mathbf{x}, t)+z^{2} \vartheta_{2}^{*}(\mathbf{x}, t)$.

Combination of the variational formulation for thermoelastic problems [13] with assumptions of the KLT for thin plates yields the strong form of the governing equations in view of the PDE:

$$
\begin{gathered}
\sum_{a=1}^{2} C^{\left(\theta \vartheta_{a}\right)} \vartheta_{S}^{*}(\mathbf{x}, t)+\sum_{a=0}^{2} G^{\left(\theta \vartheta_{a}\right)} \vartheta_{S, \beta \beta}^{*}(\mathbf{x}, t)+ \\
+\sum_{a=0}^{2} D^{\left(\theta \vartheta_{a}\right)}\left[\dot{\vartheta}_{S}^{*}(\mathbf{x}, t)+\frac{t_{1}}{T} \ddot{\vartheta}_{S}^{*}(\mathbf{x}, t)\right]+C^{(\theta u)}\left[\dot{u}_{\beta, \beta}^{*}(\mathbf{x}, t)+\frac{t_{2}}{T} \ddot{u}_{\beta, \beta}^{*}(\mathbf{x}, t)\right]+ \\
+C^{(\theta w)}\left[\dot{w}_{, \beta \beta}^{*}(\mathbf{x}, t)+\frac{t_{2}}{T} \ddot{w}_{, \beta \beta}^{*}(\mathbf{x}, t)\right]=0 \\
C^{(u u)} \tau_{\alpha \beta, \beta}^{*(u)}(\mathbf{x}, t)+\sum_{a=0}^{2} C^{\left(u \vartheta_{a}\right)} \tau_{\alpha \beta, \beta}^{*\left(\vartheta_{a}\right)}(\mathbf{x}, t)+C^{(u w)} \tau_{\alpha \beta, \beta}^{*(w)}(\mathbf{x}, t)+ \\
+D^{(u u)} \ddot{u}_{\alpha}^{*}(\mathbf{x}, t)+D^{(u w)} \ddot{w}_{, \alpha}^{*}(\mathbf{x}, t)=0 \\
C^{(w u)} \tau_{\alpha \beta, \alpha \beta}^{*(u)}(\mathbf{x}, t)+C^{(w w)} \tau_{\alpha \beta, \alpha \beta}^{*(w)}(\mathbf{x}, t)+\sum_{a=0}^{2} C^{\left(w \vartheta_{a}\right)} \tau_{\alpha \beta, \alpha \beta}^{*\left(\vartheta_{a}\right)}(\mathbf{x}, t)+ \\
+D_{1}^{(w w)} \ddot{w}^{*}(\mathbf{x}, t)-D_{2}^{(w w)} \ddot{w}_{, \beta \beta}^{*}(\mathbf{x}, t)-D^{(w u)} \ddot{u}_{\beta, \beta}^{*}(\mathbf{x}, t)=-\bar{t}_{3}^{*}(\mathbf{x}, t)
\end{gathered}
$$

plus two complementary algebraic equations should be composed from the possible thermal boundary conditions on the bottom and top of the plate given by Dirichlet type:

$$
\vartheta_{0}^{*}(\mathbf{x}, t) \pm \frac{1}{2} \vartheta_{1}^{*}(\mathbf{x}, t)+\frac{1}{4} \vartheta_{2}^{*}(\mathbf{x}, t)=\frac{\bar{\theta}(\mathbf{x}, \pm 1 / 2, t)}{\theta_{0}}-1
$$

or Neuman type:

$$
\pm k_{0} k_{H}(\mathbf{x}) k_{V}( \pm 1 / 2)\left[\vartheta_{1}^{*}(\mathbf{x}, t) \pm \vartheta_{2}^{*}(\mathbf{x}, t)\right]=\frac{\bar{q}(\mathbf{x}, \pm 1 / 2, t)}{\theta_{0}}
$$


The most frequently applied boundary conditions for mechanical fields on the boundary edge of the plate are given as follows:

(i) on clamped edge:

$$
\left.u_{\alpha}^{*}(\mathbf{x}, t)\right|_{\partial \Omega}=0,\left.w^{*}(\mathbf{x}, t)\right|_{\partial \Omega}=0,\left.\frac{\partial w^{*}}{\partial \mathbf{n}}(\mathbf{x}, t)\right|_{\partial \Omega}=0
$$

(ii) on simply supported edge:

$$
\left.n_{\beta}(\mathbf{x}) T_{\alpha \beta}^{*}(\mathbf{x}, t)\right|_{\partial \Omega}=0,\left.w^{*}(\mathbf{x}, t)\right|_{\partial \Omega}=0,\left.n_{\alpha}(\mathbf{x}) n_{\beta}(\mathbf{x}) M_{\alpha \beta}^{*(w)}(\mathbf{x}, t)\right|_{\partial \Omega}=0,
$$

while for thermal fields on the boundary edge $\partial \Omega$ we name the following boundary conditions

(i) Dirichlet type: $\left.\vartheta_{0}^{*}(\mathbf{x}, t)\right|_{\partial \Omega}=\bar{\theta}(\mathbf{x}, z=0, t) / \theta_{0}-1$

(ii) Neuman type: $-\left.k_{0} k_{H}(\mathbf{x}) k_{V}(0) n_{\beta}(\mathbf{x}) \vartheta_{0, \beta}^{*}(\mathbf{x}, t)\right|_{\partial \Omega}=\bar{q}(\mathbf{x}, 0, t) / \theta_{0}$

The deformation fields are defined as

$$
\begin{aligned}
& \tau_{\alpha \beta}^{*(u)}(\mathbf{x}, t)=\frac{H}{2}\left(u_{\alpha, \beta}^{*}(\mathbf{x}, t)+u_{\beta, \alpha}^{*}(\mathbf{x}, t)\right)+v \delta_{\alpha \beta} u_{\gamma, \gamma}^{*}(\mathbf{x}, t) \\
& \tau_{\alpha \beta}^{*(w)}(\mathbf{x}, t):=H w_{, \alpha \beta}^{*}(\mathbf{x}, t)+v \delta_{\alpha \beta} \nabla^{2} w^{*}(\mathbf{x}, t) \\
& \tau_{\alpha \beta}^{*\left(\vartheta_{a}\right)}(\mathbf{x}, t):=(1+v) \vartheta_{a}^{*}(\mathbf{x}, t) \delta_{\alpha \beta},
\end{aligned}
$$

while coefficients $C^{()}, G^{()}, D^{()}$are defined in [13].

The gradients of deformation fields include higher order derivatives of primary field variables, which lead to increasing inaccuracy of the solution. To overcome this problem, we decompose original $4^{\text {th }}$ order governing PDEs into coupled set of $2^{\text {nd }}$ order PDEs by introducing new field variables as:

$$
m^{*}(\mathbf{x}, t):=\nabla^{2} w^{*}(\mathbf{x}, t), \quad s_{\alpha}^{*}(\mathbf{x}, t):=\nabla^{2} u_{\alpha}^{*}(\mathbf{x}, t) .
$$

Then, we receive:

$$
\begin{aligned}
\tau_{\alpha \beta, \beta}^{*(u)}(\mathbf{x}, t)= & \frac{H}{2} s_{\alpha}^{*}(\mathbf{x}, t)+\frac{H+2 v}{2} u_{\beta, \beta \alpha}^{*}(\mathbf{x}, t), \quad \tau_{\alpha \beta, \alpha \beta}^{*(u)}(\mathbf{x}, t)=(H+v) s_{\beta, \beta}^{*}(\mathbf{x}, t) \\
& \tau_{\alpha \beta, \beta}^{*(w)}(\mathbf{x}, t)=(H+v) m_{, \alpha}^{*}(\mathbf{x}, t), \quad \tau_{\alpha \beta, \alpha \beta}^{*(w)}(\mathbf{x}, t)=(H+v) \nabla^{2} m^{*}(\mathbf{x}, t) \\
& \tau_{\alpha \beta, \beta}^{*\left(\vartheta_{a}\right)}(\mathbf{x}, t):=(1+v) \vartheta_{a, \alpha}^{*}(\mathbf{x}, t), \quad \tau_{\alpha \beta, \alpha \beta}^{*\left(\vartheta_{a}\right)}(\mathbf{x}, t):=(1+v) \nabla^{2} \vartheta_{a}^{*}(\mathbf{x}, t)
\end{aligned}
$$


Now, 3 equations given by (9), plus 4 equations given by (4), plus 2 complementary algebraic equations composed from the possible thermal boundary conditions on the bottom and top of the plate given by Eq. (5) yield the complete set of governing equations for the primary field variables

$$
\left\{w^{*}(\mathbf{x}, t), m^{*}(\mathbf{x}, t), u_{\beta}^{*}(\mathbf{x}, t), s_{\beta}^{*}(\mathbf{x}, t), \vartheta_{0}^{*}(\mathbf{x}, t), \vartheta_{1}^{*}(\mathbf{x}, t), \vartheta_{2}^{*}(\mathbf{x}, t)\right\} .
$$

\section{Moving Least Squares (MLS) approximation technique}

In this paper, we have used the strong formulation with employing the meshless approximation for primary field variables by Central Approximation Node (CAN) concept of Moving Least Square (MLS) approximation technique [3-4].

Without going into details [5], the approximation of field variable $u(\mathbf{x})$ around the central approximation node $\mathbf{x}^{q}$ can be expressed by

$$
u(\mathbf{x}) \approx \sum_{a=1}^{N^{q}} \hat{u}^{\bar{a}} \phi^{(q, a)}(\mathbf{x}), \quad \bar{a}=n(q, a),
$$

where $\bar{a}$ is the global number of the $a$-th node from the influence domain of $\mathbf{x}^{q}, N^{q}$ is the number of nodal points in the influence domain, and $\phi^{(q, a)}(\mathbf{x})$ is the shape function associated with the node $n(q, a)$. The CAN can be selected as the nearest node to the field point $\mathbf{x}$.

The derivatives of the field variable $u(\mathbf{x})$ can be approximated by derivatives of approximated fields (D0-approach) [3], i.e.

$$
u_{, i j k \ldots l}(\mathbf{x}) \approx \sum_{a=1}^{N^{q}} \hat{u}^{\bar{a}} \phi_{, i j k \ldots l}^{(q, a)}(\mathbf{x}) .
$$

Since the nodal unknowns are time-dependent, the spatially discretized equations are given by the system of ODE (ordinary differential equations). For numerical solution of the system of the ODE for nodal unknowns we employ the Wilson $\theta$-method [11-12] which assumes a linear change of accelerations within the time interval $\left[t_{k}, t_{k}+\theta \Delta t\right]$, where $\Delta t$ is the time step and $t_{k+1}=t_{k}+\Delta t$. Without going into details of the time stepping algorithm, remember that the Wilson $\theta$ - method is one-step method (it is not required to know the solution at two or more previous steps) and implicit method, since in order to get the solution $u^{(k+1)}=u\left(t_{k+1}\right)$ it is not sufficient to know only $u^{(k)}, \stackrel{\circ}{ }^{(k)}$ and, $\ddot{u}^{(k)}$ but it is necessary to solve also the governing equations. 


\section{Numerical experiments}

In presented numerical investigations of thin elastic plates under thermal loads within the KLT, we consider a square plate $L \times L$ with clamped all the edges, $L=1$, and in-plane gradations of material properties. Poisson's ratio is assumed to be constant $v=0.3$, while various values of the power-law gradation exponent are considered. The thermal loading is applied as: $\quad \bar{q}(\mathbf{x}, \pm h / 2, t)=0, \quad$ for $\quad \mathbf{x} \in \Omega \cup \partial \Omega, \quad \bar{q}\left(x_{1}, 0,0, t\right)=0, \quad \bar{q}\left(x_{1}, L, 0, t\right)=0$, $\bar{\theta}\left(0, x_{2}, 0, t\right)=\theta_{0}, \bar{\theta}\left(L, x_{2}, 0, t\right)=\theta_{0}+20\left(1-e^{t / \tau}\right)$ for $\mathbf{x} \in \partial \Omega$. The mechanical loading is omitted, $\bar{t}_{3}^{*}(\mathbf{x}, t)=0$, and the initial conditions are vanishing $u_{\alpha}(\mathbf{x}, t=0)=0$, $w(\mathbf{x}, t=0)=0, \vartheta_{S}(\mathbf{x}, t=0)=0$.

In all numerical computations, we have used a uniform distribution of nodal points (16 $\times 16$ nodes) with $\delta=1 / 15$ being the distance between two neighbour nodes. The other parameters in the MLS-approximation have been taken as: radius of the interpolation domain $\rho^{a}=3.001 \delta$, shape function parameter $c^{a}=\delta$, and cubic polynomial basis.

In what follows, we shall present the results for temperature, in-plane displacements, and deflections in the case of transversal gradation of material coefficients within the generalized and classical thermoelasticity. From Fig. 1, it can be seen that the transversal gradation of Young's modulus and/or thermal expansion coefficient has negligible influence on the evolution of temperature neither in case of linear nor non-linear gradation. On the other hand, the transversal gradation of material coefficients associated with heat conduction equation results into observable changes in time variation of temperatures. The linear and/or non-linear gradation of heat conduction coefficient has accelerating effect on the evolution of the temperature, while the transversal gradation of the mass density and/or specific heat capacity has an opposite effect. 

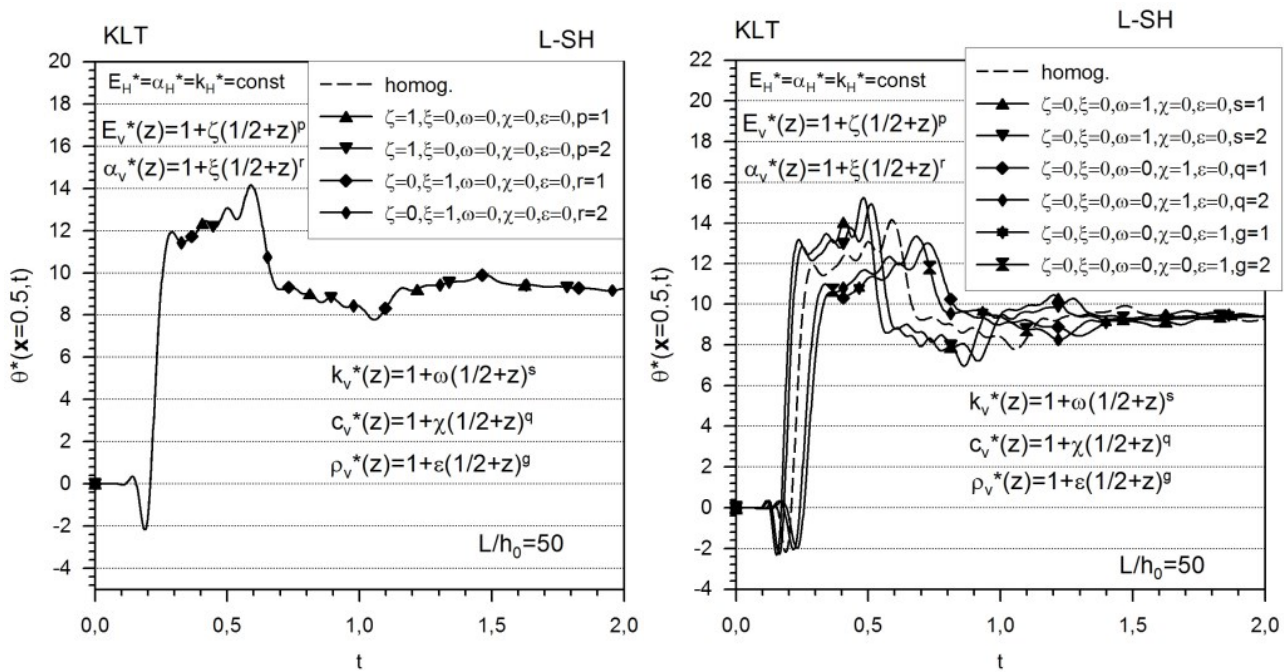

a)
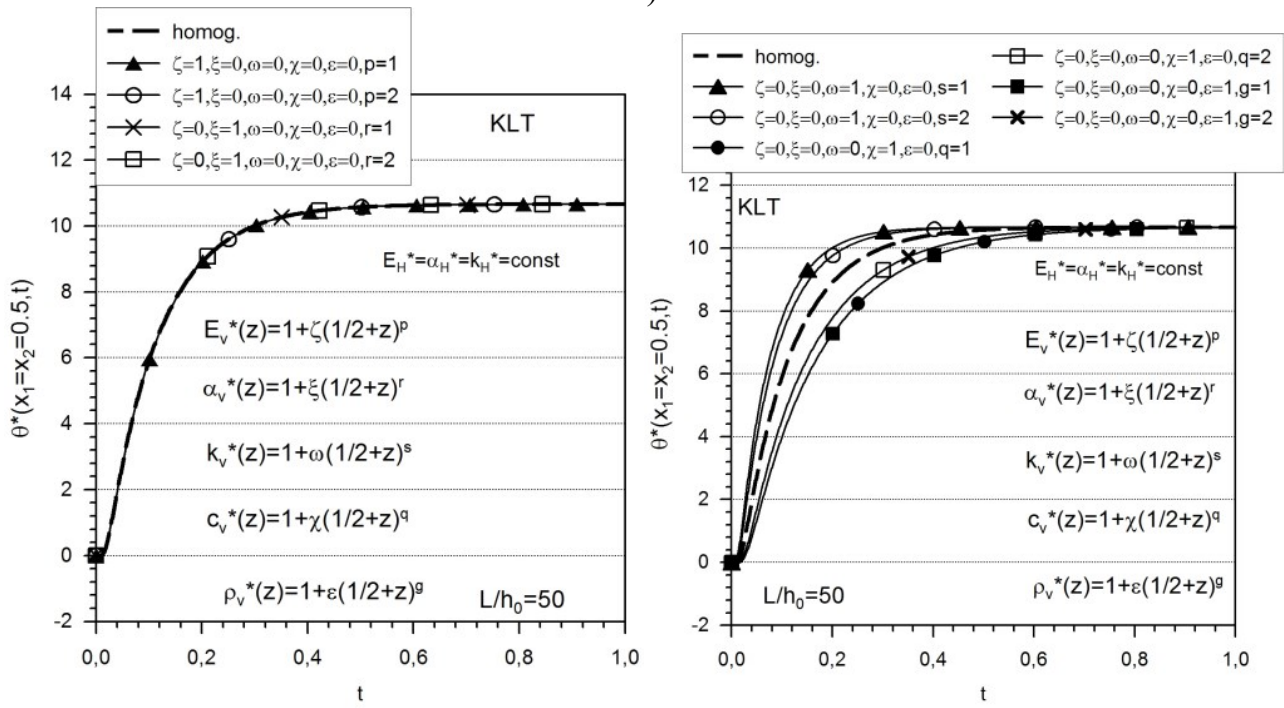

b)

Fig. 1. Time evolution of temperature within FGM plates in a) generalized (L-SH) and b)classical theory of thermoelasticity.

Contrary to the temperature, the time evolution of in-plane displacements is clearly affected by the transversal gradation of thermal expansion coefficient (Fig. 2). The influence of gradation of Young's modulus is again negligible. The influence of the transversal gradation of the heat conduction coefficient, specific heat capacity and mass density on time evolution of in-plane displacements is similar to that on temperature. Note that the steady state values of in-plane displacements and temperature are not affected, but they are affected during the transition times. 

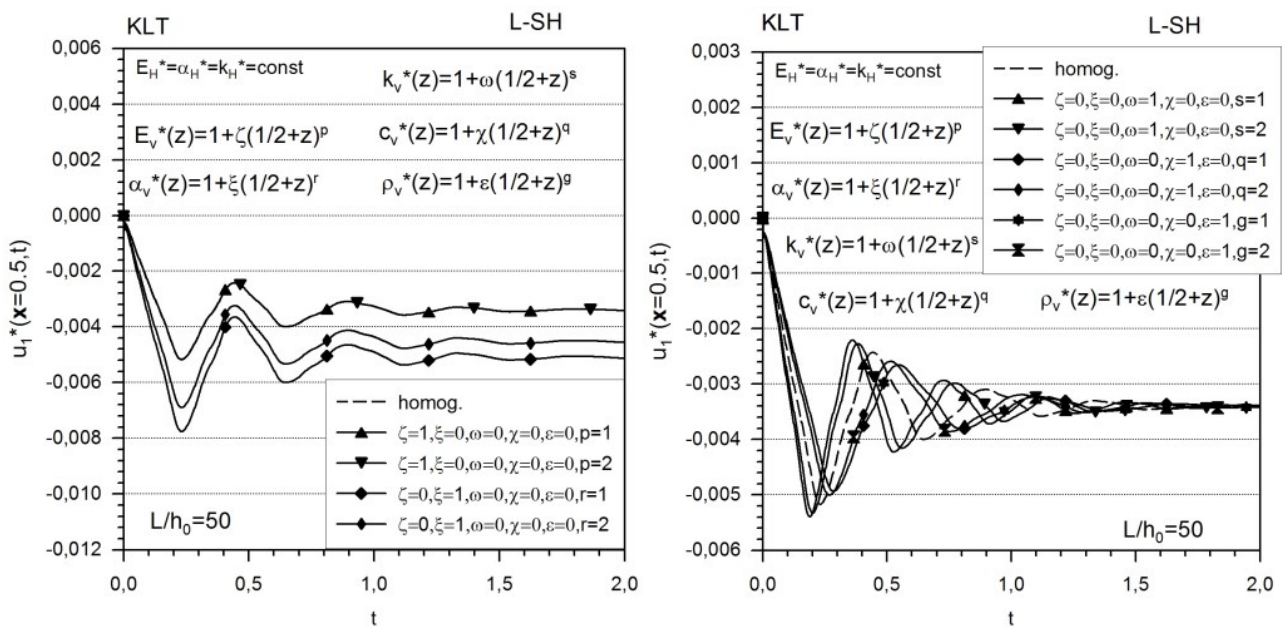

a)
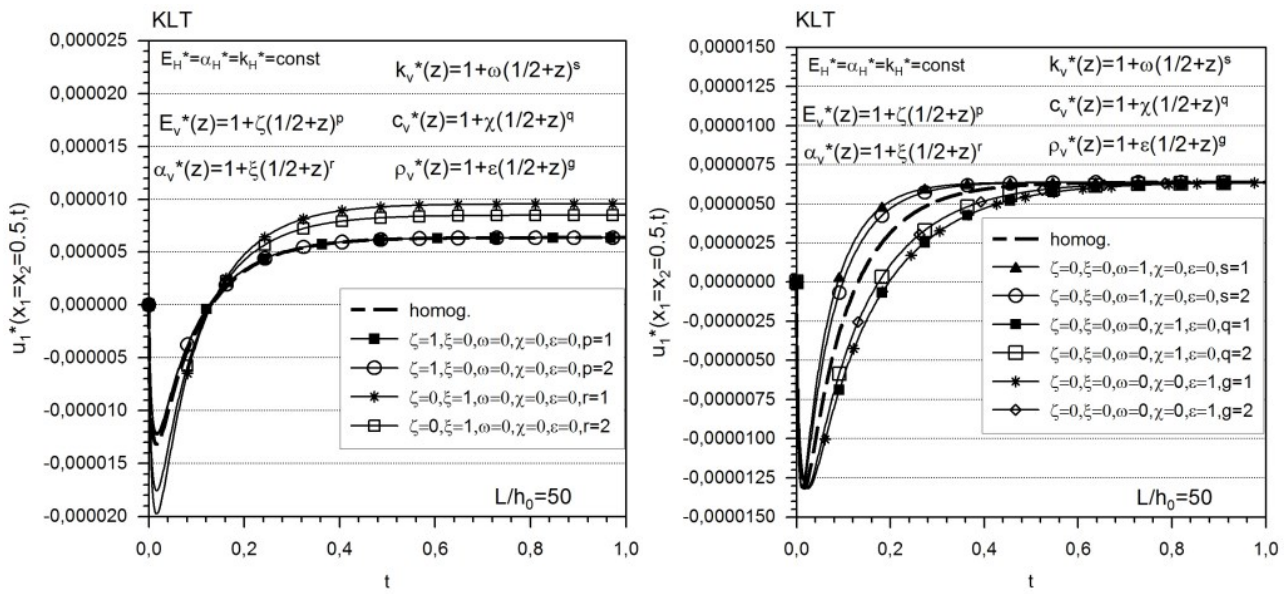

b)

Fig. 2. Time evolution of in-plane displacements within FGM plates in a) generalized (L-SH) and b) classical theory of thermoelasticity.

The time evolutions of deflections at the midpoint of the homogeneous and FGM plates are shown in Fig.3. Apparently, the transversal gradation of both the Young's modulus as well as thermal expansion coefficient gives rise to finite deflections of the plate with clamped edges. This due to the coupling between the in-plane displacements and deflection if the Young's modulus is graded in transversal direction. The gradation of the other material coefficients does not give rise finite deflections. The necessary condition for finite steady state deflections in the considered plate with clamped edges is non-uniform distribution of temperature in the mid-plane.

Note that the differences between the classical and generalized theory of thermoelasticity behaviour for time evolution of field variables are clearly visible. The wave nature of spreading of heat within the generalized theory is observable. 

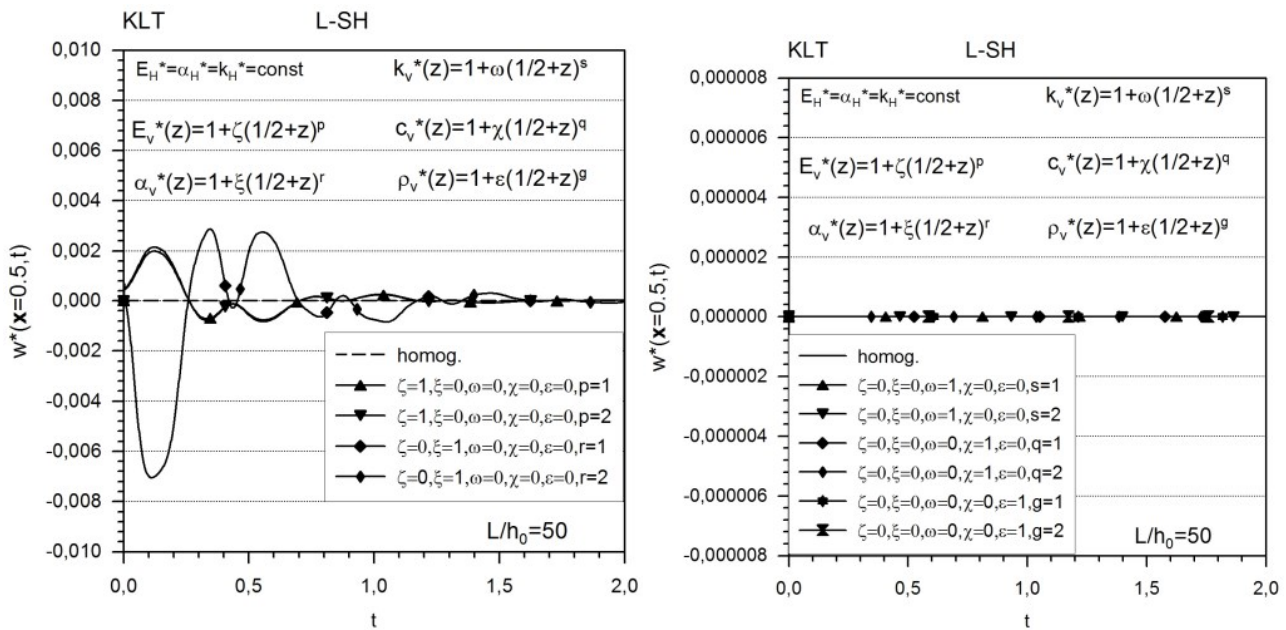

a)
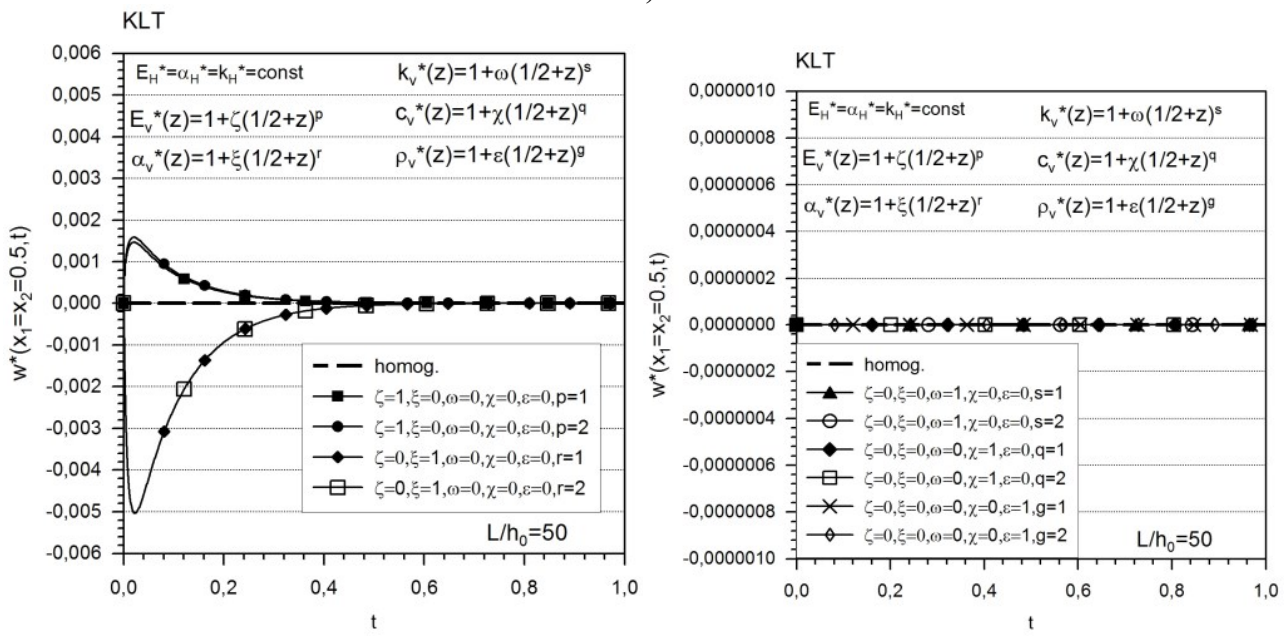

b)

Fig. 3. Time evolution of deflections within FGM plates in a) generalized (L-SH) and b)classical theory of thermoelasticity.

\section{Conclusion}

In this paper the thin plate bending problem within the classical and generalized thermoelasticity with assumptions of Kirchhoff-Love theory is presented The analysis of the derived governing equations reveals new coupling effects in the considered multi-field problem, which are not present in homogeneous plates. The functional gradation of material coefficients can bring coupling between the in-plane and bending elastic modes as well as coupling between the temperature and elastic fields. Combining the strong formulation with compactly supported meshless approximation, we developed an effective element-free method for solution of boundary value problems of FGM plates under arbitrary thermal loadings. Numerical implementation and simulations have been performed in order to study and explain the coupling effects with including the wave nature of spreading of heat in FGM plates subjected to a transient thermal loading. 
The financial support by the Slovak Research and Development Agency under the contract No. APVV-14-0440 is greatly acknowledged.

\section{References}

1. G. A.E. and L. K., Thermoelasticity. J. Elast., 2, 1-7 (1972)

2. P. Lancaster and K. Salkauskas, Surfaces generated by moving least squares method. Math. Comput., 37, 141-158 (1981)

3. V. Sladek, J. Sladek and C. Zhang, Computation of stresses in non-homogeneous elastic solids by local integral equation method: a comparative study. Computational Mechanics, 41, 827-845 (2008)

4. V. Sladek, J. Sladek and C. Zhang, Local integral equation formulation for axially symmetric problems involving elastic FGM. Engineering Analysis with Boundary Elements, 32, 1012-1024 (2008)

5. V. Sladek, J. Sladek and L. Sator, Physical decomposition of thin plate bending problems and their solution by mesh-free methods. Engineering Analysis with Boundary Elements, 37, 348-365 (2013)

6. L. Sator, V. Sladek, J. Sladek and D. Young, Elastodynamics of FGM plates by meshfree method. Composite Structures, 140, 309-322 (2016)

7. L. Sator, V. Sladek and J. Sladek, Coupling effects in elastic analysis of FGM composite plates by mesh-free methods. Composite Structures, 115, 100-110 (2014)

8. L. Sator, V. Sladek and J. Sladek, Bending analysis of FGM plates under thermal load. Procedia Engineering, 190, 54-61 (2017)

9. Vel.S.S. and B. R.C, Generalized plane strain thermoelastic deformation of laminated anisotropic thick plates. Int. J. Solids Struct, 38, 1395-1414 (2001)

10.H.W. Lord; Y.A. Shulman, A generalized dynamical theory of thermoelasticty. J. Mech. Phys. Solids, 15, 229-309 (1967)

11. E.L. Wilson, I. Farhoomand, K.J. Bathe, Nonlinear dynamic analysis of complex structures. Earthquake Eng Struct Dyn, 1, 241-252 (1973)

12. I. Gladwell, R. Thomas, Stability of the Newmark. Houbolt and Wilson $\theta$ methods. Int J Numer Anal Methods Geomech, 4, 143-158 (1980)

13. L. Sator, V. Sladek, J. Sladek, Bending of FGM plates under thermal load: Classical thermoelasticity analysis by a meshless method. Composites Part B 146 176-188 (2018) 